013 Implementing "Evaluation for Transformation" as a Conceptual Framework to Measure the Impact of the USDA Farm to School Grant Program

Matthew Benson, PhD, Matthew.Benson@fns.usda.gov, United States Department of Agriculture, 1400

Independence Avenue SW, Washington, DC 20250;

M. Russell, MNO; A. Powers, PEER Associates

Objective: This presentation describes implementing "Evaluation for Transformation" as a conceptual framework for measuring the impact of the USDA Farm to School Grant Program.

Target Audience: Target audience includes project coordinators from entities eligible for a USDA Farm to School Grant including schools, agricultural producers or producer groups, state and local agencies, nonprofit entities and Indian tribal organizations.

Theory, Prior Research, Rationale: Evaluation of the USDA Farm to School Grant Program draws on the National Farm to School Network's evaluation framework, "Evaluation for Transformation." This framework proposes standardized indicators and measures in four areas farm to school programs are thought to impact: community economic development, public health, education and the environment.

Description: On an annual basis, USDA awards up to $\$ 5$ million in Farm to School Grants to support schools improving access to local foods in school meals. In 2015, grantees $(n=55)$ completed standardized baseline and final reports which collected information about a select group of common measures in each of the four impact areas.

Evaluation: Preliminary results show that these grant projects are positively impacting each of the four impact areas. Grantees are reporting the majority of these positive impacts in the areas of community economic development, public health and education with fewer positive impacts in the area of improved environmental quality.

Conclusions and Implications: This is one of the first examples of a group implementing "Evaluation for Transformation" as a framework for evaluating the impact of farm to school programs. Implications include developing a national evidence base for farm to school programs and creating a common approach for evaluating and reporting on these activities and programs.

Funding: None

\section{Diet Quality and Characteristics Differ Among Food Secure and Food Insecure Emergency Food Pantry Users}

Heather Eicher-Miller, PhD, Purdue University, STON G2, 700 West State Street, West Lafayette, IN 47907;

L. McCormack, PhD, MPH, RD, LN, ACSM EP-C, South
Dakota State University; S. Stluka, MS, RD, LN;

D. Contreras, PhD, Michigan State University; L. FranzenCastle, MS, RD, PhD, University of Nebraska-Lincoln;

B. Henne, MS, Michigan State University; D. Mehrle, MPH, $R D, L D$, University of Missouri; D. Remley, $P h D, M S P H$, Ohio State University

Objective: Food insecurity, or uncertain access to healthy foods, is associated with various negative health outcomes, and affects roughly 70\% of the emergency food pantry (EFP)-user population. This study evaluated differences in characteristics of food secure and food insecure EFP clients, as well as the relationship between food security status and diet quality.

Design, Setting, and Participants: EFP Clients $(\mathrm{N}=599)$ from a multistate sample of 24 Midwestern, rural EFPs participated at a single time point from SeptemberNovember 2014.

Outcome Measures and Analysis: Participants completed the 18-item U.S. Household Food Security Module, and one to three automated self-administered 24-hour dietary recalls (ASA24s) that were used to calculate Healthy Eating Index-2010 (HEI) total and component scores. Chi-square analysis determined differences in characteristics between food security groups. Multiple linear regression models adjusted for confounders were used to determine associations between food security status and HEI scores.

Results: Significant differences between food security groups in age, household size, participation in food assistance programs, and use of EFPs existed. A positive association between household food security status and total dairy intake $(\beta=0.75, \mathrm{p}=0.05)$ was determined. However, no other significant associations were found.

Conclusions and Implications: Food security status may be positively associated with dairy intake in the EFPuser population, but overall lack of association between food security status and diet quality suggests that mere use of EFPs may be an indicator of poor diet quality among clients. Additional research is needed to investigate how EFP use over time may be related to changes in food security and diet quality.

Funding: NIFA

\section{Calorie Labels on the Restaurant Menu: Weight-Control Behaviors and Ordering Decisions of Young Adults}

Nicole Larson, PhD, MPH, RDN, University of Minnesota, 1300 South Second Street, Suite 300, Minneapolis, MN 55337; A. Haynos, PhD; K. Loth, PhD, MPH, RD;

D. Neumark-Sztainer, PhD, MPH, RD

Objective: This study examines associations between the use of calorie information on restaurant menus and weight-control behaviors in a population-based sample of young adults.

Continued on page $S 7$ 
015 (continued)

Design and Participants: Weight-control behaviors are being assessed as part of the fourth wave of Project EAT, a 15-year longitudinal study. Data collection using online, phone, and mailed surveys will be complete in January 2016. The preliminary dataset described here represents 1,777 survey respondents (57\% female, mean age=31).

Measures and Analysis: Participants were asked to report past-year dieting and use of six healthy (e.g., exercise) and 10 unhealthy (e.g., fasting) weight-control behaviors. Analysis to be completed in the full dataset will adjust for demographic covariates and use response weights to account for sample attrition since baseline.

Results: Results showed $57 \%$ of young adults noticed calorie information while purchasing a meal or snack at a restaurant in the past month. Among those who noticed calorie information, 60\% reported using the information to limit calorie intake by avoiding a high calorie item or deciding on a smaller portion size. Using calorie information to limit energy intake was more often reported by young adults who endorsed other weight control behaviors; however, it was not uncommon among those not did not. For example, using calorie information to limit intake was reported by $65 \%$ of young adults who endorsed one or more unhealthy weight-control behaviors and by $56 \%$ of those who did not $(\mathrm{p}=0.005)$.

Conclusions and Implications: Results suggest it will be important to conduct further research evaluating the impact of restaurant menu calorie labeling among populations with weight concerns.

Funding: National Heart Lung and Blood Institute, National Institute of Mental Health

\section{SNAP-Ed Program Characteristics Were Not Associated With Improvement in Food Security}

Heather A. Eicher-Miller, PhD, Department of Nutrition Science, Purdue University, 700 West State Street, Lafayette, IN 47907; M. K. Maulding, MS, RD;

A. R. Abbott, MA, RD, CD; Q. Wang

Objective: To determine the association of Supplemental Nutrition Assistance Program-Education (SNAP-Ed) program characteristics with long-term food security among households with children in Indiana after a SNAP-Ed intervention.

Design, Setting, and Participants: A randomized, controlled nutrition education intervention study using SNAP-Ed as the intervention was implemented across 38 Indiana counties from August 2013 to March 2015. Study participants $(n=575)$ were low-income SNAP-Ed eligible adults $\geq 18$ years with $\geq 1$ child living in the household. SNAP-Ed paraprofessionals recruited and randomized participants to the control or intervention group who received $\geq 4$ SNAP-Ed lessons over 4-10 week intervention period. All participants completed a baseline, post-intervention, and 1 year follow-up assessment.
Outcome Measures and Analysis: Household food security score was assigned using the 18-item United States Household Food Security Survey Module. Linear mixed regression models adjusted for lesson delivery format (categorical variable with 3 levels: individual, group, combination) and SNAP-Ed paraprofessional (categorical variable with each paraprofessional as a level) in addition to other household characteristics were used to compare study treatment group and program characteristic effects over time on household food security score. Results were considered significant at $\mathrm{p} \leq 0.05$.

Results: Lesson delivery format $(\mathrm{p}=0.5)$ and the individual SNAP-Ed paraprofessional $(\mathrm{p}=0.6)$ were not significantly associated with improvement in household food security from baseline to 1 year follow-up. Approximately half $(57 \%)$ of intervention group participants received lessons individually.

Conclusions and Implications: Differences in SNAPEd lesson delivery format and para-professional were not critical factors in improving food security.

Funding: University of Kentucky Center for Poverty Research, U.S. Department of Agriculture, Food and Nutrition Service, AG-3198-S-12-0044.

\section{Differing Contributions of Food Sources to Dietary Energy, Solid Fat and Added Sugar in US Adults by Food Security Status}

Christopher Taylor, PhD, RD, LDN, FAND, Ohio State University, 453 West 10th Avenue, 306 Atwell Hall, Columbus, OH 43210; N. Hooker, PhD; J. Clark, PhD; R. Watowicz, MS, RDN, LD; C. Spees, PhD, RDN, LD, FAND

Objective: Food insecurity in US adults is linked to nutritional inequality and numerous health disparities. Understanding the food environment and how it contributes to dietary patterns among food insecure adults is important to promoting healthy, balanced eating.

Design, Setting, and Participants: An analysis of the 2011-2012 National Health and Nutrition Examination Survey was conducted to assess the proportional contribution of food sources to energy, discretionary solid fats and added sugars by food security status. Dietary intakes from 4,801 adults were assessed using a 24-hour recall. The originating food source was reported and categorized into ten discreet categories. Household food security status was determined using the 18-item USDA Core Food Security Survey Module.

Outcome Measures and Analysis: The sums of dietary energy, added sugars and discretionary solid fats were aggregated from where each food was obtained. The proportions of total energy, fat and added sugars obtained from each food access point was estimated.

Results: The majority (55-62\%) of total energy, solid fats and added sugars were obtained from general retail stores across all food security categories. Fast food establishments accounted for a greater proportion of energy

Continued on page $S 8$ 